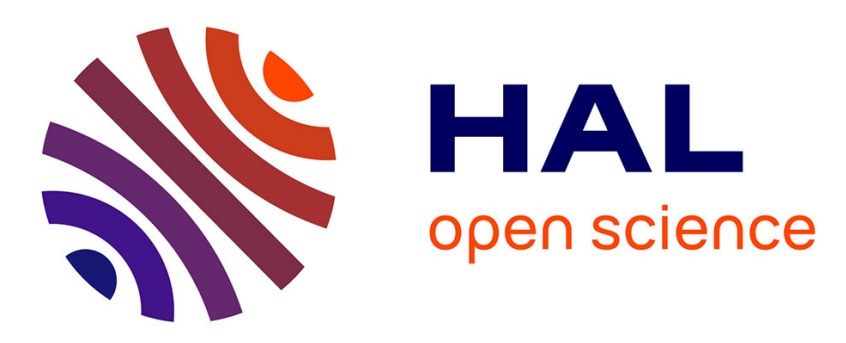

\title{
Handling Missing Values with Regularized Iterative Multiple Correspondence Analysis
}

Julie Josse, Marie Chavent, Benoit Liquet, François Husson

\section{To cite this version:}

Julie Josse, Marie Chavent, Benoit Liquet, François Husson. Handling Missing Values with Regularized Iterative Multiple Correspondence Analysis. Journal of Classification, 2012, 29 (1), pp.91-116. 10.1007/s00357-012-9097-0 . hal-00763227v3

\section{HAL Id: hal-00763227 \\ https://hal.science/hal-00763227v3}

Submitted on 19 Dec 2012

HAL is a multi-disciplinary open access archive for the deposit and dissemination of scientific research documents, whether they are published or not. The documents may come from teaching and research institutions in France or abroad, or from public or private research centers.
L'archive ouverte pluridisciplinaire HAL, est destinée au dépôt et à la diffusion de documents scientifiques de niveau recherche, publiés ou non, émanant des établissements d'enseignement et de recherche français ou étrangers, des laboratoires publics ou privés. 


\title{
Handling Missing Values with Regularized Iterative Multiple Correspondence Analysis ${ }^{1}$
}

\author{
J. Josse ${ }^{\mathrm{a} 2}$, M. Chavent ${ }^{\mathrm{b}}$, B. Liquet ${ }^{\mathrm{c}}$ and F. Husson ${ }^{\mathrm{a}}$ \\ a Agrocampus, 65 rue de St-Brieuc, 35042 Rennes, France; \\ ${ }^{\text {b} U n i v e r s i t e ́ ~ V . ~ S e g a l e n ~ B o r d e a u x ~ 2, ~} 146$ rue L. Saignat, 33076 Bordeaux, France \\ 'Equipe Biostatistique de l'U897 INSERM, ISPED
}

\begin{abstract}
A common approach to deal with missing values in multivariate exploratory data analysis consists in minimizing the loss function over all non-missing elements. This can be achieved by EM-type algorithms where an iterative imputation of the missing values is performed during the estimation of the axes and components. This paper proposes such an algorithm, named iterative multiple correspondence analysis, to handle missing values in multiple correspondence analysis (MCA). This algorithm, based on an iterative PCA algorithm, is described and its properties are studied. We point out the overfitting problem and propose a regularized version of the algorithm to overcome this major issue. Finally, performances of the regularized iterative $M C A$ algorithm (implemented in the R-package named missMDA) are assessed from both simulations and a real dataset. Results are promising with respect to other methods such as the missing-data passive modified margin method, an adaptation of the missing passive method used in Gifi's Homogeneity analysis framework.

Key words: Multiple Correspondence Analysis; Categorical Data; Missing Values; Imputation; Regularization
\end{abstract}

\section{Introduction}

Multiple correspondence analysis (MCA) is an exploratory data analysis method which allows to sum-up and to visualize a data table in which individuals are described by several categorical variables. Standard references include Benzécri (1973), Nishisato (1980), Lebart et al. (1984), Greenacre (1984), Gifi (1981) and recently Greenacre and Blasius (2006). MCA is well suited for the analysis of questionnaires to study the association among the variables. Data collected within questionnaires are often incomplete due to respondents skipping a question or refusing to answer to a question, etc. Non-responses may arise from different reasons that have to be distinguished. Indeed, the choice of a method to deal with missing values and the properties of the methods depend on the kind of missing values.

A first distinction can be done between "really missing" values and "not really missing" values (Little and Rubin, 1987, 2002). "Not really missing"

\footnotetext{
${ }^{1}$ Preprint of Journal of classification, Vol. 29, pp. 91-116.

${ }^{2}$ Corresponding author. Email: josse@agrocampus-ouest.fr
} 
means that the missing value does not mask an underlying category among the available categories of the variable. The missing value has a specific meaning and represents a new category in itself. For example, in a survey, some respondents may be unable to choose a response option for a question and a missing value may identify a "don't know" category. It then represents a new dimension in the space of the variable. "Really missing" means that the individual would have chosen one category among the available categories. For "really missing" values, Rubin (1976) has distinguished three different mechanisms that lead to missing data: missing completely at random (MCAR), missing at random (MAR), and missing not at random (MNAR). MCAR means that the probability that a value is missing is unrelated to the value itself and any values in the dataset, missing or observed. MAR means that the probability that a value is missing is unrelated to the value itself but is related to some observed values on the other variables. For example, lets consider two variables: activity (with the categories executive woman and retired) and budget for food consumption per week (with the categories less than $5 \%$ of your income, $5 \%$ to $10 \%, 10 \%$ to $15 \%, 15 \%$ to $20 \%$, more than 20\%). MAR means that the probability of missing values on budget variable depends on the activity variable; indeed, executive women may not want to respond to a question that takes too much time. However, within each type of activity the probability of missing values for the budget variable is unrelated to budget. Finally, MNAR means that the probability that a value is missing is related to the value itself. For example, missing values on the variable alcohol consumption (with the categories Not at all, less than 1 time per week, 2 or 3 times a week, 4 to 6 times a week, 7 or more than 7 times a week) may mask an embarrassment due to the extreme consumption. Schafer and Graham (2002) specify that it is difficult to know the kind of missing values. Most of the methods dealing with missing values are dedicated to MAR and MCAR values.

van der Heijden and Escofier (2003) have produced a very complete review of the various methods available to handle missing values in MCA and have discussed which method is most suited for each kind of missing data. For example, they explain that one of the most popular methods, the missing single method, which consists in creating an extra category for missing values and performing the MCA on the new dataset, is frequently used in practice for all kinds of missing data. However, according to the previous definitions, this strategy is actually more adapted to "not really missing" or MNAR values. They also detail another method named the "missing insertion-ventilation" method (Lebart et al., 1984) where a category is allocated to missing values with a random sampling among the categories with respect to their frequencies. They do not recommend this method for dealing with missing values in MCA. This latter method as well as the missing single method corresponds to the default option in many softwares. van der Heijden and Escofier (2003) highlight the good behaviour of the missing passive modified margin method proposed by Escofier (1987) and in detailed section 2.

In this paper, a new method named regularized iterative $M C A$ is proposed to deal with MCAR and MAR values. Section 2 presents MCA as a weighted principal component analysis (PCA) and details the missing passive modified 
margin method. Section 3 describes the iterative $M C A$ algorithm and gives its main properties. Then, section 4 focuses on the overfitting problem and presents the regularized iterative $M C A$ algorithm to overcome this major issue. Finally, the method is illustrated using a fictive dataset and a simulation study is conducted to compare the performances of the proposed algorithm to the wellknown methods recommended by van der Heijden and Escofier (2003). Results obtained from a real dataset are also presented.

\section{Multiple correspondence analysis}

\subsection{Presentation of MCA as a weighted PCA}

Let us consider a dataset with $I$ individuals and $J$ categorical variables $\mathbf{v}_{j}, j=$ $1, \ldots, J$ with $k_{j}$ categories. The data are coded using the indicator matrix of dummy variables, denoted $\mathbf{X}$ of size $I \times K$ with $K=\sum_{j=1}^{J} k_{j}$. MCA can be presented as the PCA of the following $(\mathbf{Z}, \mathbf{M}, \mathbf{D})$ triplet:

$$
\left(I \mathbf{X D}_{\Sigma}^{-1}, \frac{1}{I J} \mathbf{D}_{\Sigma}, \frac{1}{I} \mathbb{I}_{I}\right)
$$

with $\mathbf{D}_{\Sigma}=\operatorname{diag}\left(\left(I_{k}\right)_{k=1, \ldots, K}\right)$ the diagonal matrix of the column margins of the matrix $\mathbf{X}$. The matrix $\mathbf{D}=\frac{1}{I} \mathbb{I}_{I}$ (with $\mathbb{I}_{d}$ corresponding to the identity matrix of size $d$ ) corresponds to the row masses and the matrix $\mathbf{M}=\frac{1}{I J} \mathbf{D}_{\Sigma}$ is the metric (used to compute distances between rows).

More precisely, performing PCA of a triplet $(\mathbf{Z}, \mathbf{M}, \mathbf{D})$ consists in the following singular value decomposition (SVD):

$$
\mathbf{Z}=\mathbf{C} \Lambda \mathbf{U}^{t},
$$

where

$$
\mathbf{C}^{t} \mathbf{D C}=\mathbf{U}^{t} \mathbf{M U}=\mathbb{I}_{r} .
$$

The $I \times r$ matrix $\mathbf{C}$ is the matrix of eigenvectors of $\mathbf{Z} \mathbf{M} \mathbf{Z}^{t} \mathbf{D}$ in descending order of the $r$ largest eigenvalues, $\mathbf{U}$ is the $K \times r$ matrix of eigenvectors of $\mathbf{Z}^{t} \mathbf{D Z M}$ in descending order of the $r$ largest eigenvalues, $\Lambda$ is a diagonal matrix with singular values (of the matrices $\mathbf{Z M Z} \mathbf{Z}^{t} \mathbf{D}$ and $\mathbf{Z}^{t} \mathbf{D Z M}$ ) on the diagonal, in weakly descending order, and $r$ is the rank of $\mathbf{Z}$. In MCA, there are at most $r=$ $K-J$ non zero eigenvalues. The columns of $\mathbf{C}$ correspond to the standardized principal components and we note $\mathbf{F}=\mathbf{C} \Lambda$ the principal components (the scores or the individual coordinates). The columns of $\mathbf{U}$ correspond to the axes (the loadings). Note that in MCA the first singular value and the corresponding singular vectors are trivial. Indeed the first singular value is one and the first columns of $\mathbf{C}$ and $\mathbf{U}$ are respectively $\mathbf{1}_{I}$ and $\mathbf{1}_{K}$ (an $I \times 1$ and $K \times 1$ vector of ones). The others singular values and vectors could then be obtained by the SVD of the centred matrix of $\mathbf{Z}: \mathbf{Z}-\mathbf{1}_{I} \mathbf{m}$ with $\mathbf{m}$ the mean vector of the columns of $\mathbf{Z}$.

A lower rank approximation approach. Let $\|\mathbf{A}\|_{\mathbf{M}, \mathbf{D}}=\sqrt{\operatorname{tr}\left(\mathbf{A} \mathbf{M} \mathbf{A}^{t} \mathbf{D}\right)}$ the Hilbert-Schmidt norm. MCA provides the best low rank $S<r$ approximation 
of the matrix $\mathbf{Z}$ in the least square sense. Indeed, by selecting the $S$ largest singular values and the corresponding singular vectors in the previous SVD, MCA gives $\mathbf{F}_{I \times S}$ and $\mathbf{U}_{K \times S}$ that minimize the reconstruction error criterion:

$$
\mathcal{C}=\left\|\mathbf{Z}-\mathbf{F} \mathbf{U}^{t}\right\|_{\mathbf{M}, \mathbf{D}}^{2}
$$

The best approximation of the matrix $\mathbf{Z}$ by a matrix of $\operatorname{rank} S$ is $\hat{\mathbf{Z}}=\hat{\mathbf{F}} \hat{\mathbf{U}}^{t}$. The approximation of the indicator matrix is then $\hat{\mathbf{X}}=\frac{1}{I} \hat{\mathbf{Z}} \mathbf{D}_{\Sigma}$. Tenenhaus and Young (1985) has checked that $\hat{\mathbf{X}}$ has the margins of $\mathbf{X}$ and that each sub-matrix $\hat{\mathbf{X}}_{j}$, associated to each variable $\mathbf{v}_{j}(j=1, \ldots, J)$, has the margins (columns and rows) of $\mathbf{X}_{j}$. This lower rank approximation approach is the foundation of the iterative $M C A$ method proposed to handle missing values (see section 3 ).

A constrained optimization approach. Each component $\mathbf{f}_{s}$ (the $s$-th column of the matrix $\mathbf{F}$ ) with variance $\lambda_{s}$ is also the solution of an optimisation problem:

$$
\hat{\mathbf{f}}_{s}=\underset{f_{s} \in \mathbb{R}^{I}}{\arg \max } \sum_{j=1}^{J} \hat{\eta}_{\mathbf{f}_{s} \mid \mathbf{v}_{j}}^{2}
$$

under the constraints that $\mathbf{f}_{s}$ is orthogonal to $\mathbf{f}_{t}$ with $1 \leq t<s$ and $\hat{\eta}_{\mathbf{f}_{s} \mid \mathbf{v}_{j}}^{2}$ is the sample correlation ratio between the $j$-th categorical variable $\mathbf{v}_{j}$ and the continuous variable $\mathbf{f}_{s}$.

\subsection{Missing passive modified margin method to deal with missing values}

Meulman (1982) detailed a method named missing passive to deal with missing data in the framework of homogeneity analysis (Gifi, 1981; Michailidis and de Leeuw, 1998; Takane and Oshima-Takane, 2003). This method is based on the following assumption: if an individual $i$ has not answered to the variable $j$, one considers that the individual has not chosen any category for the variable. Consequently, in the indicator matrix, the entries in the row corresponding to individual $i$ and variable $j$ are marked 0 . It leads to row margins that are not equal to the number of variables $J$. This strategy has several shortcomings. When the row margins are not equal to the same constant (the number of missing values may be different from one individual to another), many properties of MCA are lost (Escofier, 1987; van der Heijden and Escofier, 2003). For example, denoting $x_{i}$. and $x_{i^{\prime}}$. the row margin for the row $i$, respectively $i^{\prime}$, the distance between the two individuals is:

$$
d\left(i, i^{\prime}\right)=\sum_{k=1}^{K}\left(\frac{x_{i k}}{x_{i .}}-\frac{x_{i^{\prime} k}}{x_{i^{\prime} .}}\right)^{2} \frac{I K}{I_{k}} .
$$

Consequently, even if the individuals $i$ and $i^{\prime}$ have chosen the same category $k$ for one variable, the quantity $\left(\frac{x_{i k}}{x_{i}}-\frac{x_{i^{\prime} k}}{x_{i^{\prime}}}\right)$ is different from 0 if their row margins are not equal and as a consequence the distance between the two individuals is 
increased which is inappropriate. In this formulae as well as in many others, some simplifications can't be performed which leads to some property loss.

Escofier (1987) has then developed the method missing passive modified margin method to overcome this problem. It consists in substituting the row margins of the indicator matrix by $J$ for all the calculations of MCA. Compared to the missing passive method, the same indicator matrix (with rows of 0) is used but the metrics and masses change. The missing passive modified margin method has the interesting property that each component $\mathbf{f}_{s}$ maximises $\sum_{j=1}^{J} \hat{\eta}_{\mathbf{f}_{s} \mid \mathbf{v}_{j}}^{2}$ under the additional constraint that $\mathbf{f}_{s}$ is forced to be orthogonal to the constant vector. In this sense, this method "skips" the missing values since an individual which has not answered to item $j$ is ignored for variable $j$.

van der Heijden and Escofier (2003) have suggested that missing passive modified margin method is suited for MCAR and MAR values. From our point of view, this method does allow to skip the missing informations but it seems more appropriate for dealing with "not really missing" values. Indeed, keeping rows of 0 for the missing values boils down to consider that individuals have not taken any of the available categories and may have taken an additional one. This point of view is strengthened by the presentation of the subset MCA method proposed by Greenacre and Pardo (2006). Subset MCA is a method whereby a sub-cloud of points (a subset of categories) can be studied with the metrics of the complete cloud of points. Greenacre and Pardo (2006) propose to use this method in the framework of missing values. They consider that a missing value represents a potential category and codes it as a new category. However, they explain that it frequently leads to results dominated by the missing values (all the missing categories are towards the periphery of the graph). The subset MCA method allows focusing on the observed categories and neglecting the missing ones. It is thus a way to clearly visualize the results without being disturbed by

the missing values. In addition, the subset MCA method in the framework of missing values gives exactly the same results than the missing passive modified margin method. As such, it comforts our feelings about Escofier's method. This method can be seen as a method that creates a new category for missing values without having to visualize it. Moreover no attempt is made to extract information from the nonresponses.

\section{Iterative MCA}

In this section we propose a new approach named iterative $M C A$ to handle missing values in MCA. This method considers that missing values mask underlying values and consequently is mainly devoted to MCAR or MAR values. The objective of the algorithm is to obtain the MCA axes and components in spite of the missing values. 


\subsection{Iterative MCA algorithm}

An approach commonly used to deal with missing values in exploratory data analysis methods such as PCA, consists in ignoring the missing values by minimizing the reconstruction error over all non-missing elements. This can be achieved by introducing a weight matrix $\mathbf{W}$ (with $w_{i k}=0$ if $z_{i k}$ is missing and $w_{i k}=1$ otherwise) in the least square criterion:

$$
\mathcal{C}=\left\|\mathbf{W} *\left(\mathbf{Z}-\mathbf{F} \mathbf{U}^{t}\right)\right\|_{\mathbf{M}, \mathbf{D}}^{2},
$$

with $*$ the Hadamard product. In contrast to the complete case, there is no explicit solution to minimize the criterion (2) and it is necessary to resort to iterative algorithms. It is possible to use weighted criss-cross multiple regression algorithm (Gabriel and Zamir, 1979) or algorithms in the spirit of the EM one (Dempster et al., 1977). The core of this latter algorithm consists in setting the missing elements at initial values, performing the analysis (such as PCA or CA) on the completed dataset, updating the missing values with the reconstruction formulae using a predefined number of components and repeating the procedure on the newly obtained matrix until the total change in the matrix falls below an empirically determined threshold. Such algorithms has been first proposed in the framework of correspondence analysis by Nora-Chouteau (1974); Greenacre (1984) and the iterative PCA algorithm has been detailed in PCA by Kiers (1997) and Josse et al. (2009). In joint correspondence analysis (Greenacre, 1988; Greenacre and Blasius, 2006), such algorithms are used to fit only the non-diagonal part of the Burt matrix. This allows obtaining percentages of variability that are less pessimistic than in MCA.

Since MCA has been presented as the PCA of the triplet $(\mathbf{Z}, \mathbf{M}, \mathbf{D})$, an iterative MCA algorithm can then be defined. The methodology to deal with missing values in PCA is extended to MCA but the algorithm is adapted to take into account the specificities of MCA. The iterative PCA algorithm is used but the metric $\mathbf{M}$ is updated during the estimation process since it depends on the data table. Indeed, after imputing data with the reconstruction formulae, the column margins of the new data table change.

If an individual $i$ has a missing value for item $j$, it leads to a row of missing values in the indicator matrix $\mathbf{X}$ for the variable $j$. The procedure is then carried out according to the following steps:

1. initialization $\ell=0$ : calculate the indicator matrix $\mathbf{X}^{0}$ and substitute initial values to missing values (for example, affect the proportion of the category using the non-missing entries). $\mathbf{X}$ can have noninteger entries but the sum of the entries corresponding to one individual and one variable must equal one.

Calculate the margins of the completed indicator matrix: the margin of each row is equal to the number of variables (i.e. $J$ ), the margin of column $k$ is equal to $I_{k}^{0}$ the sum of column $k$. Calculate the matrix $\mathbf{D}_{\Sigma}^{0}=$ $\operatorname{diag}\left(\left(I_{k}^{0}\right)_{k=1, \ldots, K}\right)$

2. step $\ell$ : 
(a) perform the MCA on the completed matrix $\mathbf{X}^{\ell-1}$, it means the PCA on the triplet

$$
\left(I \mathbf{X}^{\ell-1}\left(\mathbf{D}_{\Sigma}^{\ell-1}\right)^{-1}, \frac{1}{I J} \mathbf{D}_{\Sigma}^{\ell-1}, \frac{1}{I} \mathbb{I}_{I}\right)
$$

to obtain $\hat{\mathbf{F}}^{\ell}$ and $\hat{\mathbf{U}}^{\ell}$; these parameters are obtained with the singular value decomposition of $\left(I \mathbf{X}^{\ell-1}\left(\mathbf{D}_{\Sigma}^{\ell-1}\right)^{-1}-\mathbf{1}_{I} \mathbf{1}_{K}^{\prime}\right) \times \sqrt{\frac{\mathbf{D}_{\Sigma}^{\ell-1}}{I J}}$;

(b) keep the first $S$ dimensions and use the reconstruction formulae to compute:

$$
\hat{\mathbf{Z}}^{\ell}=\left(\mathbf{1}_{I} \mathbf{1}_{K}^{\prime}+\left(\hat{\mathbf{F}} \hat{\mathbf{U}}^{\prime}\right)^{\ell} \times \sqrt{\frac{I J}{\mathbf{D}_{\Sigma}^{\ell-1}}}\right) .
$$

Calculate the associated values in the indicator matrix using the margins of step $\ell-1$

$$
\hat{\mathbf{X}}^{\ell}=\frac{1}{I} \hat{\mathbf{Z}}^{\ell} \mathbf{D}_{\Sigma}^{\ell-1}
$$

and the new imputed dataset is $\mathbf{X}^{\ell}=\mathbf{W} * \mathbf{X}+(1-\mathbf{W}) * \hat{\mathbf{X}}^{\ell}$

(c) the column margins $I_{k}^{\ell}$ of the new completed matrix $\mathbf{X}^{\ell}$ are calculated and gathered in $\mathbf{D}_{\Sigma}^{\ell}$;

3. steps (2.a), (2.b) and (2.c) are repeated until the change in the imputed matrix falls below a threshold $\left(\sum_{i k}\left(\hat{x}_{i k}^{\ell-1}-\hat{x}_{i k}^{\ell}\right)^{2} \leq \varepsilon\right.$, with $\varepsilon$ equal to $10^{-6}$ for example).

In the imputation step (step 2.b), missing values are imputed in such a way that they do not contribute to the reconstruction error. In this sense, missing values are also said to be "skipped".

Remark. The initialization step consists in performing the missing fuzzy average method. This method is equivalent to the mean imputation method for continuous variables. It consists in substituting the proportion of each category to the missing entries in the indicator matrix. It also corresponds to imputation with the reconstruction formulae with $S=0: \hat{x}_{i k}=\frac{I_{k}}{I}$. An interesting property of this method is that the imputed values do not contribute to the total inertia (total variance). Indeed, missing values are imputed by the average profile and the total inertia measures the gap to independence. In this sense, imputed values have no influence on the solution and missing values are also said to be "skipped". This method also named "reconstruction of order 0" is used in the framework of correspondence analysis (de Leeuw and van der Heijden, 1988).

\subsection{Properties}

\subsubsection{Barycentric relations}

At each step of the algorithm, within the completed indicator matrix all the observed cases take 0 and 1 values whereas the imputed cases (corresponding to 
the missing values) are real numbers. A very convenient property of this fuzzy matrix is that its margin per variable is still 1 even with the imputed values $\left(\forall i, \forall j, \quad \sum_{k=1}^{K_{j}} \hat{x}_{i k}=1\right)$. Consequently, all the main properties of MCA are preserved such as barycentric relations.

Indeed, it can be shown that for all $i$ and for all $j, \sum_{k=1}^{K_{j}} x_{i k}^{\ell}=1$ if $\sum_{k=1}^{K_{j}} x_{i k}^{\ell-1}=1$. Using the reconstruction formulae of step 2.b of the iterative MCA algorithm, we can write:

$$
\begin{aligned}
\sum_{k=1}^{K_{j}} \hat{x}_{i k}^{\ell} & =\sum_{k=1}^{K_{j}} \frac{1}{I}\left(1+\sum_{s=1}^{S} \hat{f}_{i s}^{\ell} \hat{u}_{k s}^{\ell} \sqrt{\frac{I J}{I_{k}^{\ell-1}}}\right) I_{k}^{\ell-1}, \\
& =\sum_{k=1}^{K_{j}} \frac{I_{k}^{\ell-1}}{I}+\frac{\sqrt{J}}{\sqrt{I}} \sum_{k=1}^{K_{j}} \sum_{s=1}^{S} \hat{f}_{i s}^{\ell} \hat{u}_{k s}^{\ell} \sqrt{I_{k}^{\ell-1}}, \\
& =\frac{\sum_{k=1}^{K_{j}} I_{k}^{\ell-1}}{I}+\frac{\sqrt{J}}{\sqrt{I}} \sum_{s=1}^{S} \hat{f}_{i s}^{\ell}\left(\sum_{k=1}^{K_{j}} \hat{u}_{k s}^{\ell} \sqrt{I_{k}^{\ell-1}}\right) .
\end{aligned}
$$

If we have $\sum_{k=1}^{K_{j}} \hat{x}_{i k}^{\ell-1}=1$ then $\sum_{k=1}^{K_{j}} I_{k}^{\ell-1}=I$ and each set of categories has a weighted average at the origin of the map, i.e. $\sum_{k=1}^{K_{j}} u_{k s}^{\ell} \sqrt{I_{k}^{\ell-1}}=0$ for all $s$. Consequently, the last (right) term of equation (3) is equal to zero and for all $j$ and for all $i \sum_{k=1}^{K_{j}} \hat{x}_{i k}^{\ell}=1$.

\subsubsection{Imputation}

Even if the objective of the algorithm is to obtain the MCA axes and components, the indicator matrix is imputed during the estimation steps. Based on the axes and components, this imputation takes into account the similarities between individuals and the relationships between variables. The iterative $M C A$ method improves the prediction of the missing values compared to missing fuzzy average method (which is the first step in the algorithm) in the same way that the regression imputation improves the mean imputation for continuous data. An imputed value can be seen as a "degree of membership" for the corresponding category. The missing entries of the original dataset may be imputed to the most plausible category.

\subsubsection{Starting values}

As usual in iterative algorithms, the final solution is sensitive to the initialization parameters and it may be interesting to explore different initial values. However, for all initial values, the row margin per variable should be equal to 1 in order to ensure the barycentric relations as demonstrated in the previous section. 


\subsubsection{Number of components}

The choice of the number of components used for the imputation step (step 2.b) is crucial and is done a priori. If the number of components is insufficient, relevant information may be forgotten in the analysis. An excessive number of components is also problematic since noise is taken into account and consequently the results are unstable. Methods to select the number of components in the incomplete case are thus required. Several strategies such as permutation tests (Takane and Hwang, 2002) allow choosing the dimensionality in MCA in the complete case, but they cannot be easily extended to the incomplete case. On the contrary, the EM cross-validation method, as the one proposed in the framework of complete PCA by Bro et al. (2008), can be extended to select the number of components in a missing data framework. Since the number of dimensions affects the prediction of the imputed values and the estimation of the axes and components, the mean square error of prediction (MSEP) appears to be a well-fitted criterion to select the number of components. For a fixed $S$, it consists in removing each observed value alternatively (leave-one out) from the original dataset and predicting the indicator matrix using the iterative $M C A$ algorithm. One missing value in the original dataset for individual $i$ and variable $j$ generates several missing values in the indicator matrix (missing values for all the categories of the variable $j$, i.e. for $x_{i k}, k=\left\{1, \ldots, k_{j}\right\}$ ). Then, the quantity $\left(x_{i k}-\hat{x}_{i k}^{-i k}\right)$ is computed for all elements $\{i k\}$, with $\hat{x}_{i k}^{-i k}$ the predicted value for the cell $\{i k\}$ calculated from the dataset without $x_{i k}, k=\left\{1, \ldots, k_{j}\right\}$. It leads to a matrix of prediction errors and the MSEP is calculated by:

$$
\frac{1}{I J} \sum_{i=1}^{I} \sum_{j=1}^{J} \sum_{k=1}^{k_{j}}\left(x_{i k}-\hat{x}_{i k}^{-i k}\right)^{2} .
$$

The number $S$ that leads to the smallest MSEP is retained.

\section{Regularized iterative MCA}

This section focuses on the overfitting problem which is the main problem of the iterative $M C A$ algorithm. A regularized version of the previous algorithm is then proposed to overcome this major issue.

\section{$4.1 \quad$ Overfitting}

Overfitting means that the value of the criterion (2) is low, i.e. fit is good on the observed values, whereas the quality of prediction of the missing values is very poor due to a bad estimate of the axes and components. Overfitting problems may occur when many parameters are estimated with respect to the number of observed values. This problem is especially important when the number of missing values is high and the number of dimensions kept for the reconstruction is high. Overfitting problems may also occur when the structure of the dataset is low, meaning that the relationships between variables are not strong. Let 
us consider an example to illustrate these problems with 100 individuals, 10 variables and a strong structure (the simulation process is detailed in the simulation section). The two-dimensional configuration associated to this dataset is given figure 1 on the left. Then, $10 \%$ of values are removed and the iterative $M C A$ algorithm is performed on this incomplete dataset. The fitting error (corresponding to the criterion (2)) and the error of prediction (using the matrix $1-\mathbf{W}$ rather than $\mathbf{W}$ in the criterion $(2))$ are quite close (0.027 versus 0.057$)$. The configuration obtained with the iterative $M C A$ algorithm (not presented here) is very similar to the true one. Consequently, with $10 \%$ missing values there is no overfitting problem in this case. However, when more values are removed (leading for example to $30 \%$ missing values) the iterative MCA algorithm encounters difficulties. Indeed, with $30 \%$ missing values, the fitting error is low (0.024) whereas the prediction error is nearly 5 times higher $(0.119)$, which is characteristic of the overfitting problem. Within the MCA configuration, the overfitting problem results in points (individuals and categories) that are very far from each other (see figure 1 in the middle). This is very frequent when datasets have many missing values. In the same way, overfitting is exacerbated with a low structure even if the percentage of missing values is small.

A first way to reduce overfitting is to reduce the number of dimensions for the imputation step in order to estimate less parameters; however it is important not to remove too many components since information can be lost. Another solution commonly used to overcome overfitting problems is to resort to shrinkage methods. Such methods have been mainly described in regression frameworks (Hastie et al., 2001). For example, the ridge estimator (Hoerl and Kennard, 1970 ) is slightly biased but has a smaller variance than the least square estima-

tor. Consequently, the mean squared error of the parameters (squared bias plus variance) is often better with a ridge regression than with an ordinary regression. Further, a ridge regression gives better predictions. In the framework of MCA, the same principles apply: the estimation of the parameters (axes and components) and the prediction of the missing values obtained by the iterative $M C A$ algorithm may have a very high variance whereas a regularized version of this algorithm stabilizes these predictions. In the next section, such an algorithm is presented.

\subsection{Regularized iterative MCA algorithm}

The regularized algorithm is quite similar to the iterative $M C A$ one but a "shrunk" reconstruction step is substituted to the classic reconstruction step (step 2.b). With $\lambda_{s}$ the eigenvalue of rank $s$, step (2.b) can be rewritten as:

$$
\left(\hat{z}_{i k}^{\ell}-1\right) \sqrt{\frac{I_{k}^{\ell-1}}{I J}}=\left(\sum_{s=2}^{S} \frac{\hat{f}_{i s}^{\ell}}{\left\|\hat{\mathbf{f}}_{s}^{\ell}\right\|_{\mathbf{D}}}\left(\sqrt{\lambda_{s}}\right) \hat{u}_{k s}^{\ell}\right)
$$


This step is then replaced by:

$$
\left(\hat{z}_{i k}^{\ell}-1\right) \sqrt{\frac{I_{k}^{\ell-1}}{I J}}=\left(\sum_{s=2}^{S} \frac{\hat{f}_{i s}^{\ell}}{\left\|\hat{\mathbf{f}}_{s}^{\ell}\right\|_{\mathbf{D}}}\left(\sqrt{\lambda_{s}}-\frac{\hat{\sigma}^{2}}{\sqrt{\lambda_{s}}}\right) \hat{u}_{k s}^{\ell}\right),
$$

with $\sigma^{2}$ estimated by the mean of the last eigenvalues: $\hat{\sigma}^{2}=\frac{1}{K-J-S} \sum_{s=S+1}^{K-J} \lambda_{s}$. This new algorithm named regularized iterative $M C A$ is based on the algorithm proposed in Josse et al. (2009) and Ilin and Raiko (2010) to perform PCA on an incomplete dataset. The regularized term derives from a probabilistic formulation of PCA (Tipping and Bishop, 1999). The rationale of this algorithm is to remove the noise to avoid instability on the predictions. Implicitly, it is assumed that the first dimensions contain both information and noise whereas the last ones are restricted to noise. That is why the noise variance is estimated by the mean of the last eigenvalues. Each dimension is shrunk and the regularization term (in equation 4) is well-fitted since the smallest singular values are more shrunk than the firsts. In the extreme cases when there is no noise ( $\sigma$ is equal to zero), the regularized iterative $M C A$ algorithm is equivalent to the iterative $M C A$ algorithm. At the opposite, when the noise is very important, the right hand term in equation (4) is close to 0 , consequently, $\hat{z}_{i k}$ is close to 1 and $\hat{x}_{i k}$ is close to $\frac{I_{k}}{I}$ for all $i$ and $k$. It corresponds to an imputation with the proportion of each category. This behaviour is satisfactory since there is no information in the dataset. The regularization then remains to shrink the coordinates of the individuals towards the origin.

This new algorithm is thus a way to avoid overfitting. It can be illustrated by performing the algorithm on the examples of the overfitting section. When there is no overfitting problem (in the example with a strong structure and $10 \%$ missing values), the regularized algorithm gives roughly the same results than the non regularized version (the fitting error is within the same magnitude than the prediction error and the graphical outputs are quite similar). However, when the overfitting problem occurs (in the examples with a low structure or with a high percentage of missing values), the graphical outputs as well as the associated errors are more convincing. More precisely, with the strong structure and $30 \%$ missing values, the fitting error and the prediction error are approximately of the same magnitude (0.026 versus 0.056$)$ and there are no points distant from the centre of gravity in the MCA map (figure 1 on the right). The MCA map is very close to the true configuration (figure 1 on the left).

Remark 1. The regularized algorithm can be seen as a mix between a principal components regression and a ridge regression. Indeed, in the former, the last components (corresponding to the smallest eigenvalues) are omitted for the analysis whereas in the latter all the eigenvalues are shrunk with a larger amount of shrinkage for the last dimensions (Hastie et al., 2001). In the regularized iterative $M C A$ algorithm, there is a double shrinkage: the last components are omitted and the subsequent eigenvalues are shrunk.

Remark 2. Takane and Hwang (2006) have proposed a regularized version of MCA for complete datasets to better estimate the parameters (in terms of 
mean squared errors). They explain that regularization is all the more effective when MCA is performed on datasets with small to moderate sample size or on datasets containing categories with small frequencies. It reinforces the idea that the regularization is crucial for incomplete datasets. Indeed, when there are missing values in a dataset, this means less data and less information which can be seen as a particular case of datasets with small sample size.

\section{Results}

In this section, the main methods available to deal with missing data in MCA are assessed on a toy dataset, on simulations and on a real dataset.

\subsection{A toy dataset}

To illustrate the behaviour of the regularized iterative $M C A$ as well as the other methods, we use a toy dataset with 9 individuals and 4 categorical variables $X, Y, Z, T$ (table 1 ). The first four individuals take the first category of the

\begin{tabular}{|c|ccccccccc|}
\hline var\ind & 1 & 2 & 3 & 4 & 5 & 6 & 7 & 8 & 9 \\
\hline$X$ & $X_{a}$ & $X_{a}$ & $X_{a}$ & $X_{a}$ & $X_{b}$ & $X_{b}$ & $X_{b}$ & $X_{b}$ & $X_{b}$ \\
$Y$ & $Y_{a}$ & $Y_{a}$ & $Y_{a}$ & $Y_{a}$ & $Y_{b}$ & $Y_{b}$ & $Y_{c}$ & $Y_{c}$ & $Y_{c}$ \\
$Z$ & $Z_{a}$ & $Z_{a}$ & $Z_{a}$ & $Z_{a}$ & $Z_{b}$ & $Z_{c}$ & $Z_{b}$ & $Z_{c}$ & $Z_{c}$ \\
$T$ & $T_{a}$ & $T_{b}$ & $T_{a}$ & $T_{b}$ & $T_{a}$ & $T_{b}$ & $T_{a}$ & $T_{b}$ & $T_{a}$ \\
\hline
\end{tabular}

Table 1: Toy dataset (individuals are in columns).

variables $X, Y$, and $Z$. The other individuals take other categories for these three variables and there is no link between these categories. The fourth variable $T$ is very different from the others. The two dimensional configuration of the MCA obtained from this dataset is represented on the top left figure 2 . Categories $X_{a}, Y_{a}$ and $Z_{a}$ are superimposed as well as the individuals 2 and 4 , respectively 1 and 3 . Some elements are then removed for the individuals $(1,2)$ for the variables $X$ and $Y$ (represented in grey in figure 2). Missing values are inserted on the categories which are highly linked to better spotlight the differences between methods.

The missing single, missing passive modified margin, missing fuzzy average, iterative $M C A$, and regularized iterative $M C A$ methods are performed. For the two iterative MCA algorithms, one dimension has been used (in the imputation step) since the cross-validation algorithm on the incomplete dataset suggests one dimension. The two dimensional configuration obtained by the missing single method (top right) is very different from the configuration obtained with the complete dataset. Indeed, the introduction of a new category (here NA for not available) for the missing values notably modifies the distances between individuals and generates a specific dimension (the second dimension). 
The map obtained with the missing fuzzy average method (middle left) shows that individuals 1 and 2 get closer to the centre of gravity. Indeed their missing values are imputed with the proportion and an individual in the indicator matrix which would have values equal to the proportion for each category would be at the centre of gravity on the MCA map. The map obtained with the missing passive modified margin method (middle right) is quite similar to the missing fuzzy average one. The missing passive modified margin method considers that individuals with missing values for a categorical variable have not taken any available categories. Consequently, individual 1 for example only takes categories $Z_{a}$ and $T_{a}$ and is at the barycentre of these two categories on the map.

The solution of the iterative $M C A$ algorithm is very close to the true configuration. The rationale of the method is that the link between the categories $X_{a}$, $Y_{a}$ and $Z_{a}$ is learned with individuals 3 and 4; consequently since individuals 1 and 2 take the category $Z_{a}$, it is implicitly more plausible that they take the categories $X_{a}$ and $Y_{a}$ (the indicator matrix is then imputed with values close to 1). The iterative $M C A$ algorithm provides a perfect fit. Since the links between variables are in that example perfect, the prediction is thus very good. However, finding the best fit (minimizing the fitting error equation (2)) may lead to overfitting problems when the links between variables are not perfect or when there are many missing values. Indeed, the method believes in link even if it is artificial which may lead to bad predictions.

The configuration obtained with the regularized iterative $M C A$ algorithm (bottom right) is between the iterative $M C A$ one and the missing fuzzy average one. This behaviour is expected because of the regularization term (equation (4)). Consequently, the method takes into account the links between categories (as the iterative MCA algorithm) but the regularization get the individuals slightly closer to the centre of gravity. In a way, the algorithm does not believe entirely in the links between variables which is often a good behaviour. The method balances between overfit (iterative $M C A$ ) and underfit (missing fuzzy average).

In this example, the iterative MCA algorithm lead to better results than the regularized algorithm, but is is only because the relationship between variables are perfect. In all other situations the regularization algorithm is the best one.

\subsection{Simulation studies}

A simulation study was conducted to compare the missing single, the missing passive modified margin, the missing fuzzy average and the regularized iterative $M C A$ methods. The performances of these methods are assessed from different simulated datasets with varying parameters: the percentage of missing values (small and medium), the kind of missing values (MCAR and MAR), the pattern of missing values either random or non-random (for non-random, some individuals do not answer to a set of questions), the relationships between variables (low and strong). For each set of parameters, 1000 simulations are drawn.

The RV coefficient (Escoufier, 1973; Josse et al., 2008) is used to assess the performances of the different methods. The RV coefficient is a correlation 
coefficient between two matrices which allows to compare the relative positions of the objects from one configuration to another. A modified version of the RV coefficient has been proposed (Smilde et al., 2009) in order to better interpret this coefficient. Indeed under the null hypothesis of independence between the two configurations, the expectation of the RV coefficient is not equal to 0 and depends on the number of rows and on the structure of the dataset. The modified RV coefficient eliminates this effect and its expectation is equal to 0 when there is no relationship between the two data tables.

\subsubsection{Protocol of simulation}

More precisely, the datasets are built using the following procedure:

- A dataset with 100 individuals and 10 variables is drawn from a multivariate normal distribution with a two-block diagonal covariance matrix; one block has size $6 \times 6$ and the other $4 \times 4$. The same level of correlation is used for each block, 0.4 for the low structure and 0.8 for the strong structure.

- Each variable is distributed in three equal-count categories.

- MCAR case: $10 \%$ and 30\% missing values are inserted either at random (it corresponds to the random pattern) or in the following way (corresponding to the non-random pattern): there are missing values for the first 60 individuals on the first 3 variables and for the last 60 individuals on variables 9 and 10 . For both pattern, such missing values are MCAR because they do not depend on any values (missing or not).

- MAR case: missing values are inserted in variables 2 to 6 when the variable 1 takes the first category and for variables 8 to 10 when the variable 7 takes the last category. $8 \%$ or $16 \%$ missing values are inserted either with a random pattern or not.

The number of underlying dimensions of the simulated dataset is by construction equal to four (since there are two independent blocks of variables and three categories per variable, i.e. two dimensions for each block). The cross-validation algorithm also suggests four dimensions.

\subsubsection{Behaviour of the iterative algorithms}

First, let us assess the behaviour of the two iterative algorithms namely the iterative $M C A$ (denoted iMCA) algorithm as well as the regularized iterative $M C A$ (denoted RiMCA) algorithm. Table 2 presents, within the first two columns, the number of times (over 1000 simulations) that the algorithms do not reach a solution before 1000 iterations. The regularized iterative MCA always reaches a minimum before 1000 iterations whereas the iterative MCA algorithm encounters difficulties. In these cases, the number of iterations varies between 1000 and 25000. Such situations correspond to overfitted solutions where some 
points (individuals or categories) are more distant from the centre of gravity as illustrated in figure 1.

Table 2: Convergence properties of the algorithms iMCA and RiMCA

\begin{tabular}{|c|c|c|c|c|c|c|c|}
\hline & \multirow[b]{2}{*}{ Pattern } & \multirow[b]{2}{*}{ Missing } & \multirow[b]{2}{*}{ Link } & \multicolumn{2}{|c|}{$\begin{array}{c}\text { Number of times } \\
\text { the algorithm does not converge } \\
\text { before } 1000 \text { iterations }\end{array}$} & \multicolumn{2}{|c|}{$\begin{array}{c}\text { Mean number of iterations } \\
\text { (when the algorithm converges } \\
\text { before } 1000 \text { iterations) }\end{array}$} \\
\hline & & & & iMCA.4 & RiMCA.4 & iMCA. 4 & RiMCA.4 \\
\hline 1 & $\mathrm{R}$ & $10 \%$ MCAR & low & 7 & 0 & 70 & 26 \\
\hline 2 & $\mathrm{R}$ & $10 \%$ MCAR & strong & 3 & 0 & 25 & 16 \\
\hline 3 & $\mathrm{R}$ & $30 \%$ MAR & low & 445 & 0 & 443 & 110 \\
\hline 4 & $\mathrm{R}$ & $30 \%$ MAR & strong & 314 & 0 & 265 & 68 \\
\hline 5 & NR & $10 \%$ MCAR & low & 87 & 0 & 122 & 24 \\
\hline 6 & NR & $10 \%$ MCAR & strong & 5 & 0 & 34 & 18 \\
\hline 7 & NR & $30 \%$ MAR & low & 638 & 0 & 538 & 76 \\
\hline 8 & NR & $30 \%$ MAR & strong & 187 & 0 & 252 & 61 \\
\hline 9 & $\mathrm{R}$ & $8 \%$ MAR & low & 46 & 0 & 109 & 24 \\
\hline 10 & $\mathrm{R}$ & $8 \%$ MAR & strong & 3 & 0 & 34 & 18 \\
\hline 11 & $\mathrm{R}$ & $16 \%$ MAR & low & 123 & 0 & 430 & 55 \\
\hline 12 & $\mathrm{R}$ & $16 \%$ MAR & strong & 46 & 0 & 269 & 39 \\
\hline 13 & NR & $8 \%$ MAR & low & 446 & 0 & 516 & 34 \\
\hline 14 & NR & $8 \% \mathrm{MAR}$ & strong & 109 & 0 & 192 & 38 \\
\hline 15 & NR & $16 \% \mathrm{MAR}$ & low & 188 & 0 & 476 & 74 \\
\hline 16 & NR & $16 \%$ MAR & strong & 115 & 0 & 416 & 66 \\
\hline
\end{tabular}

The last two columns of this table give the means of the number of iterations when the algorithms have converged in less than 1000 iterations. This highlights the fact that the regularized algorithm is always the fastest.

The iterative $M C A$ algorithm encounters many problems, is very slow to converge and often converges to overfitted solutions. Consequently the results of this method are not presented hereafter.

\subsubsection{Results of the simulations}

Table 3 (resp. table 4) gives the results for the configuration of the individuals (resp. categories). For each set of parameters, the modified RV coefficient is computed between the initial two-dimensional configuration (without missing values) and the two-dimensional configurations obtained by each method. The median over the 1000 modified RV coefficients is retained. The letter R corresponds to the random pattern of missing values whereas NR corresponds to the non-random pattern of missing values. For the missing single method (NA), there are only the results for individuals.

The boxplots of two particular sets of parameters with important differences between the medians (rows 8 and 12 of table 3 ) are given figure 3 . We observe that in these two cases (30\% MCAR, strong structure, random pattern and $16 \%$ MAR, strong structure, random pattern) the variability of the results for the different methods are quite small and very similar. This remark remains true for the other rows of table 3 . Consequently the medians in table 3 can be safely interpreted.

Concerning the individuals (table 3 ), the performances of the missing single method (NA) are very poor for each parameter set and especially for nonrandom patterns (NR) since distances between individuals are highly affected.

For all the other methods, the results are satisfying for a small percentage of missing values. As expected, the performances decrease when the percentage 


\begin{tabular}{llllrrrrr}
\hline & Pattern & Missing & Link & Passive & Average & NA & RiMCA.2 & RiMCA.4 \\
\hline 1 & $\mathrm{R}$ & $10 \%$ MCAR & low & 0.94 & 0.94 & 0.87 & 0.94 & 0.94 \\
2 & $\mathrm{R}$ & $10 \%$ MCAR & strong & 0.97 & 0.97 & 0.96 & 0.98 & 0.98 \\
3 & $\mathrm{R}$ & $30 \%$ MCAR & low & 0.76 & 0.76 & 0.67 & 0.76 & 0.72 \\
4 & $\mathrm{R}$ & $30 \%$ MCAR & strong & 0.88 & 0.88 & 0.86 & 0.91 & 0.92 \\
\hline 5 & $\mathrm{NR}$ & $10 \%$ MCAR & low & 0.91 & 0.92 & 0.46 & 0.93 & 0.92 \\
6 & $\mathrm{NR}$ & $10 \%$ MCAR & strong & 0.94 & 0.95 & 0.67 & 0.97 & 0.98 \\
7 & $\mathrm{NR}$ & $30 \%$ MCAR & low & 0.43 & 0.77 & 0.30 & 0.78 & 0.73 \\
8 & $\mathrm{NR}$ & $30 \%$ MCAR & strong & 0.71 & 0.91 & 0.45 & 0.90 & 0.90 \\
\hline 9 & $\mathrm{R}$ & $8 \%$ MAR & low & 0.94 & 0.94 & 0.72 & 0.95 & 0.95 \\
10 & $\mathrm{R}$ & $8 \%$ MAR & strong & 0.96 & 0.96 & 0.96 & 0.98 & 0.99 \\
11 & $\mathrm{R}$ & $16 \%$ MAR & low & 0.86 & 0.82 & 0.50 & 0.87 & 0.85 \\
12 & $\mathrm{R}$ & $16 \%$ MAR & strong & 0.88 & 0.84 & 0.88 & 0.94 & 0.96 \\
\hline 13 & $\mathrm{NR}$ & $8 \%$ MAR & low & 0.91 & 0.90 & 0.28 & 0.92 & 0.92 \\
14 & $\mathrm{NR}$ & $8 \%$ MAR & strong & 0.91 & 0.90 & 0.54 & 0.96 & 0.97 \\
15 & $\mathrm{NR}$ & $16 \%$ MAR & low & 0.80 & 0.79 & 0.28 & 0.83 & 0.79 \\
16 & $\mathrm{NR}$ & $16 \%$ MAR & strong & 0.79 & 0.77 & 0.54 & 0.89 & 0.91 \\
\hline
\end{tabular}

Table 3: Median over 1000 replications of the modified RV coefficient between the true configuration of individuals and the configurations of individuals obtained with each method; Passive for missing passive modified margin, Average for the missing fuzzy average, NA for missing single and RiMCA for the regularized iterative $M C A$ methods with 2, and 4 dimensions; $\mathrm{R}$ for random pattern and NR for non-random.

\begin{tabular}{llllrrrr}
\hline & Pattern & Missing & Link & Passive & Average & RiMCA.2 & RiMCA.4 \\
\hline 1 & $\mathrm{R}$ & $10 \%$ MCAR & low & 0.97 & 0.97 & 0.97 & 0.96 \\
2 & $\mathrm{R}$ & $10 \%$ MCAR & strong & 0.99 & 0.99 & 0.99 & 0.99 \\
3 & $\mathrm{R}$ & $30 \%$ MCAR & low & 0.85 & 0.87 & 0.84 & 0.81 \\
4 & $\mathrm{R}$ & $30 \%$ MCAR & strong & 0.95 & 0.96 & 0.95 & 0.95 \\
\hline 5 & $\mathrm{NR}$ & $10 \%$ MCAR & low & 0.96 & 0.96 & 0.96 & 0.96 \\
6 & $\mathrm{NR}$ & $10 \%$ MCAR & strong & 0.98 & 0.98 & 0.99 & 0.99 \\
7 & $\mathrm{NR}$ & $30 \%$ MCAR & low & 0.37 & 0.72 & 0.81 & 0.76 \\
8 & $\mathrm{NR}$ & $30 \%$ MCAR & strong & 0.76 & 0.85 & 0.92 & 0.92 \\
\hline 9 & $\mathrm{R}$ & $8 \%$ MAR & low & 0.97 & 0.97 & 0.97 & 0.97 \\
10 & $\mathrm{R}$ & $8 \%$ MAR & strong & 0.99 & 0.98 & 0.99 & 0.99 \\
11 & $\mathrm{R}$ & $16 \%$ MAR & low & 0.90 & 0.90 & 0.92 & 0.90 \\
12 & $\mathrm{R}$ & $16 \%$ MAR & strong & 0.95 & 0.92 & 0.95 & 0.97 \\
\hline 13 & $\mathrm{NR}$ & $8 \%$ MAR & low & 0.96 & 0.97 & 0.97 & 0.96 \\
14 & $\mathrm{NR}$ & $8 \%$ MAR & strong & 0.97 & 0.98 & 0.98 & 0.99 \\
15 & $\mathrm{NR}$ & $16 \%$ MAR & low & 0.89 & 0.91 & 0.91 & 0.86 \\
16 & $\mathrm{NR}$ & $16 \%$ MAR & strong & 0.91 & 0.92 & 0.93 & 0.93 \\
\hline
\end{tabular}

Table 4: Median over 1000 replications of the modified RV coefficient between the true categories configuration and the configurations of individuals obtained with each method; Passive for missing passive modified margin, Average for the missing fuzzy average and RiMCA for the regularized iterative $M C A$ with 2 , and 4 dimensions; $\mathrm{R}$ for random pattern and $\mathrm{NR}$ for non-random.

of missing values increases.

The performances of the missing passive modified margin algorithm (Pas- 
sive) and the missing fuzzy average method (Average) are quite similar in most situations. One can remark that in MAR situation, the missing fuzzy average method (Average) gives very poor results. This behaviour is expected because it doesn't take into account the relationships between the variables in the imputation.

The regularized iterative $M C A$ method with four dimensions RiMCA.4 is the most stable across the different patterns of missing values and the different percentages of missing values. The regularized method always provides good results even if in 4 cases it doesn't give the best ones. The other methods provide good results in many situations (except the single missing method which is not well fitted for such missing data) but sometimes they can really crash. That is a strong argument in favour of the regularized iterative $M C A$ method. The four situations (rows 3, 7, 11, and 15) when the algorithm does not provide the best results can be explained as follows. It corresponds to situations where the link is low and the percentage of missing values is important. In these four cases the regularized iterative $M C A$ method with two dimensions RiMCA.2 takes the lead. This behaviour may be explained since with low structures and many missing values, the instability increases with the number of dimensions. The estimation of the individual configurations is then better with two dimensions than with four dimensions. This behaviour is understandable. Indeed, in such situations the underlying four-dimensional structure is too low and may have disappeared. The last dimensions are not sufficiently strong and are not very stable. Consequently, taking less dimensions is a way to stabilize the predictions. From a practical point of view, the number of underlying dimensions is unknown and when the data contains many missing values and a low structure, taking few dimensions in the RiMCA algorithm should be preferred by the user. The results obtained for the categories (table 4) are quite similar to the results obtained for the individuals.

\subsection{Real data analysis}

A user satisfaction survey of pleasure craft operators on the "Canal des Deux Mers", located in South of France, was carried out by the public corporation "Voies Navigables de France" responsible for managing and developing the largest network of navigable waterways in Europe. Pleasure craft operators were asked their opinion about numerous questions with categorical answers, each item having two or three categories of response. 1232 individuals answered 14 questions with a total of 35 categories. There is $9 \%$ missing values in this dataset which concerns $42 \%$ of respondents. The data are available in the $\mathrm{R}$ package missMDA (Husson and Josse, 2010).

Figure 4 gives the two-dimensional maps of the categories and of the individuals obtained by the missing single (NA) method and the missing passive modified margin (Passive) one. The plot of the categories for the missing single (NA) method (figure 4, top left) is dominated by the missing categories (denoted for instance Q2_NA, for the non-response to question 2). The pattern of missing values, i.e. the associations between non-responses to certain questions, can be 
visualized (for instance non-response to 'Q4' is associated to non-response to 'Q5' and 'Q6'). Such pattern frequently arises in questionnaires when individuals do not answer to set of items. These missing values can be MAR, MCAR or MNAR because they can depend on other variables and it does not affect the kind of missing values. For example, missing values are MCAR if some respondents have skipped the last items in the questionnaire due to time constraints. However, it may also represent a particular behaviour and a sub-population of respondents.

The plot of the categories for the missing passive modified margin (Passive) method (figure 4, bottom left) "skips" the missing values and avoids the drawback of the NA method where the first dimensions are dominated by the missing values.

Figure 5 gives the plots of the categories and the individuals in the plane 1-2 obtained with the iterative $M C A$ and the regularized iterative $M C A$ methods. The plot of the individuals for the iterative $M C A$ method (figure 5, bottom right) highlights the overfitting problem already presented in section 4.1 (some individuals are distant from the others). The plot of the individuals for the regularized iterative $M C A$ method (figure 5, top right) confirms the importance of the regularization to reduce this phenomenon.

Note that in this real data example, the results obtained with the missing passive modified margin (Passive) and the iterative MCA methods are very similar. This is not in contradiction with the results of the simulations where the performances of the two methods were very similar in many situations especially with small percentage of missing values.

\section{Discussion}

This paper proposes the regularized iterative $M C A$ algorithm to handle missing values in MCA which is a major issue especially when dealing with questionnaires. The proposed algorithm is a regularized version of an EM-type algorithm where missing values are filled in with the expected values (via the reconstruction formulae of order $S$ ) in the expectation step and the axes and components are obtained during the maximization step. The regularization is crucial because it limits the overfitting problem. When missing values mask underlying values among the available categories and are MAR or MCAR, regularized iterative $M C A$ gives slightly better results than the other methods. This is particularly true for MAR values and when data are structured, i.e. when there are strong relationships between variables. However, this method has some drawbacks: convergence problems may occur due to the iterative nature of the algorithm and it is necessary to choose a tuning parameter (the number of components). This number is chosen by a cross-validation algorithm.

The regularized iterative $M C A$ is implemented in the $\mathrm{R}$ package ( $\mathrm{R}$ Development Core Team, 2010) missMDA (Husson and Josse, 2010). To perform MCA with missing values two steps are required. First, the function imputeMCA performs the regularized algorithm and gives as an output a completed indicator 
matrix. Then this completed indicator matrix is used as an input of the multiple correspondence analysis function MCA of the FactoMineR package (?Husson et al., 2011) to obtain the classical outputs of MCA (graphs, scores, loadings.)

MCA is sometimes used as a preprocessing step before clustering methods. If the missing single method is used, it may lead to distances between individuals disturbed by the missing entries. The regularized iterative $M C A$ is well-fitted since its main objective is to predict the coordinates of the individuals on the first components in spite of the missing values. This strategy is then a way to perform clustering on incomplete categorical variables.

It may be interesting to assess the performances of the regularized iterative $M C A$ algorithm as an imputation algorithm and to compare it to several approaches dealing with nonresponses in categorical variables. A model often used for imputation of categorical variables is the log-linear model (Schafer, 1997). However, the log-linear model can only be used for a small number of variables since it requires to compute all the entries of the multi-way cross table. Other procedures have been proposed especially in the context of the item response theory (IRT) but they are mainly devoted to dichotomous and ordinal data rather than to categorical ones. Recently, Vermunt et al. (2008) have proposed the use of the latent class model to impute large datasets. The regularized iterative $M C A$ algorithm might be competitive since it can be applied on large datasets and uses both similarities between individuals and relationships between variables for the imputation.

\section{References}

J-P. Benzécri. L'analyse des données. Tome II: L'analyse des correspondances. Dunod, 1973.

R. Bro, K. Kjeldahl, A. K. Smilde, and H. A. L. Kiers. Cross-validation of component model: a critical look at current methods. Anal Bioanal Chem, 390:1241-1251, 2008.

J de Leeuw and P G M van der Heijden. Correspondence analysis of incomplete contingency tables. Psychometrika, 53:223-233, 1988.

A P. Dempster, N M. Laird, and D B. Rubin. Maximum likelihood from incomplete data via the em algorithm. Journal of the Royal Statistical Society B, 39:1-38, 1977.

B. Escofier. Traitement des questionnaires avec non réponse, analyse des correspondances avec marges modifiée et analyse multicanonique avec contrainte. Publications de l'institut de statistique de l'université de Paris, 32:33-70, 1987.

Yves Escoufier. Le traitement des variables vectorielles. Biometrics, 29:751-760, 1973. 
K.R. Gabriel and S. Zamir. Lower rank approximation of matrices by least squares with any choice of weights. Technometrics, 21:236-246, 1979.

A. Gifi. Non-linear Multivariate Analysis. D.S.W.O.-Press, Leiden, 1981.

Michael Greenacre. Theory and Applications of Correspondence Analysis. Acadamic Press, 1984.

Michael Greenacre. Correspondence analysis of multivariate categorical data by weighted least-squares. Biometrika, 75:457-477, 1988.

Michael Greenacre and J Blasius. Multiple Correspondence Analysis and Related Methods. Chapman \& Hall/CRC, 2006.

Michael Greenacre and R. Pardo. Subset correspondence analysis: visualizing relationships among a selected set of response categories from a questionnaire survey. Sociological methods and research, 35 (2):193-218, 2006.

T Hastie, R Tibshirani, and J Friedman. The elements of statistical learning. Data Mining, Inference and Prediction. Springer series in statistics, 2001.

A F Hoerl and R W Kennard. Ridge regression: Biased estimation for nonorthogonal problems. Technometrics, 12:55-67, 1970.

Francois Husson and Julie Josse. missMDA: Handling missing values with/in multivariate data analysis (principal component methods), 2010. URL http://www.agrocampus-ouest.fr/math/husson, http://www.agrocampus-ouest.fr/math/josse. R package version 1.2.

Francois Husson, Julie Josse, Sebastien Le, and Jeremy Mazet. FactoMineR: Multivariate Exploratory Data Analysis and Data Mining with R, 2011. URL http://factominer.free.fr, http://www.agrocampus-ouest.fr/math/. $\mathrm{R}$ package version 1.16 .

A Ilin and T. Raiko. Practical approaches to principal component analysis in the presence of missing values. Journal of Machine Learning Research, page To appear, 2010.

$\mathrm{J}$ Josse, J Pagès, and F Husson. Testing the significance of the rv coefficient. Computational Statistics and Data Analysis, 53:82-91, 2008.

J Josse, J Pagès, and F Husson. Gestion des données manquantes en analyse en composantes principales. Journal de la Société Française de Statistique, 150: 28-51, 2009.

H A L Kiers. Weighted least squares fitting using ordinary least squares algorithms. Psychometrika, 62:251-266, 1997.

L Lebart, A Morineau, and K M Werwick. Multivariate Descriptive Statistical Analysis. Wiley, New-York, 1984. 
R J A Little and D B Rubin. Statistical Analysis with Missing Data. Wiley series in probability and statistics, New-York, 1987, 2002.

J Meulman. Homgeneity Analysis of Incomplete Data. D.S.W.O.-Press, Leiden, 1982.

G Michailidis and J de Leeuw. The gifi system of descriptive multivariate analysis. Statistical Science, 13:307-336, 1998.

S Nishisato. Analysis of Categorical Data: Dual Scaling and its Applications. University of Toronto Press, Toronto, 1980.

$\mathrm{C}$ Nora-Chouteau. Une méthode de reconstitution et d'analyse de données incomplètes. PhD thesis, Université Pierre et Marie Curie, 1974.

R Development Core Team. R: A Language and Environment for Statistical Computing. R Foundation for Statistical Computing, Vienna, Austria, 2010. URL http://www.R-project.org/. ISBN 3-900051-07-0.

D B Rubin. Inference and missing data. Biometrika, 63:581-592, 1976.

J L Schafer. Analysis of Incomplete Multivariate Data. Chapman \& Hall/CRC, 1997.

J L Schafer and J W Graham. Missing data: Our view of the state of the art. Psychological Methods, 7:147-177, 2002.

A K Smilde, H A L Kiers, S Bijlsma, C M Rubingh, and M J van Erk. Matrix correlations for high-dimensional data: the modified RV-coefficient. Bioinformatics, 25:401-405, 2009.

Y Takane and H Hwang. Generalized constrained canonical correlation analysis. Multivariate Behavioral Research, 37:163-195, 2002.

Y Takane and H Hwang. Regularized multiple correspondence analysis. In $\mathrm{J}$ Blasius and M J Greenacre, editors, Multiple Correspondence Analysis and Related Methods, pages 259-279. Chapman \& Hall, 2006.

Y Takane and Y Oshima-Takane. Relationships between two methods for dealing with missing data in principal component analysis. Behaviormetrika, 30:145154, 2003.

M Tenenhaus and F W Young. An analysis and synthesis of multiple correspondence analysis, optimal scaling, dual scaling, homogeneity analysis and other methods for quantifying categorical multivariate data. Psychometrika, 50:91-119, 1985.

M Tipping and C M Bishop. Probabilistic principal component analysis. Journal of the Royal Statistical Society B, 61:611-622, 1999. 
P.G.M. van der Heijden and B Escofier. Multiple correspondence analysis with missing data. In Analyse des correspondances. Presse universitaire de Rennes, 2003.

J K Vermunt, J R van Ginkel, L A van der Ark, and K Sijtsma. Multiple imputation of incomplete categorical data using latent class analysis. Sociological Methodology, 33:369-397, 2008. 


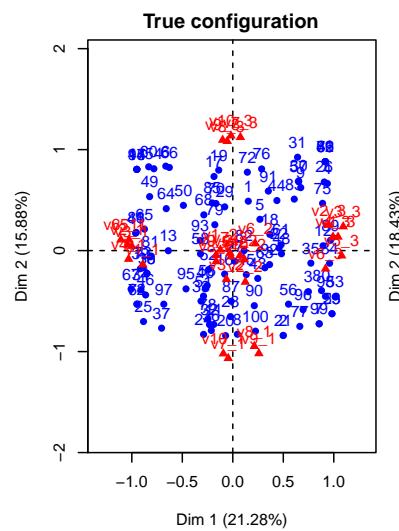

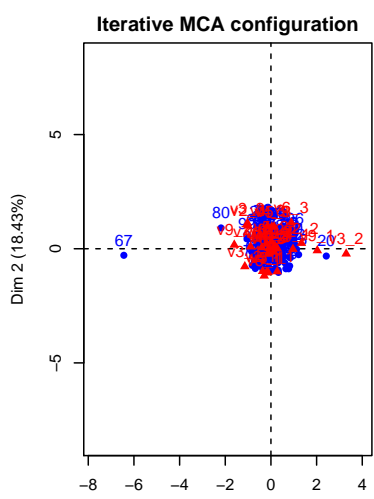

$\operatorname{Dim} 1$ (32.91\%)

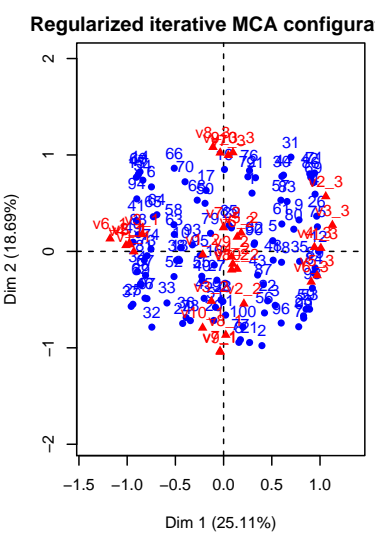

$\operatorname{Dim} 1(25.11 \%)$

Figure 1: Illustration of the overfitting problem on the MCA map obtained on a dataset with a strong structure and $30 \%$ missing values. The true configuration is on the left, the configuration obtained with the iterative $M C A$ algorithm is in the middle, the configuration obtained with the regularized iterative $M C A$ algorithm is on the right. 

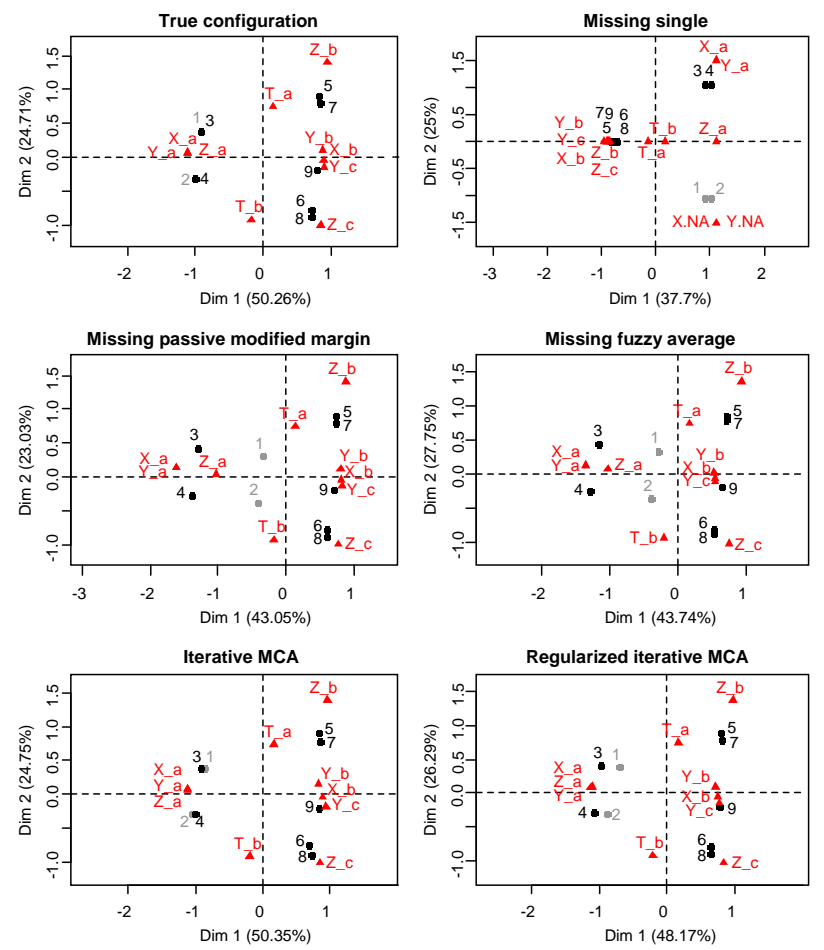

Figure 2: Comparison of the missing single, missing passive modified margin, missing fuzzy average, iterative $M C A$, and regularized iterative $M C A$ methods to handle missing values in MCA. In grey, the individuals with missing values. 

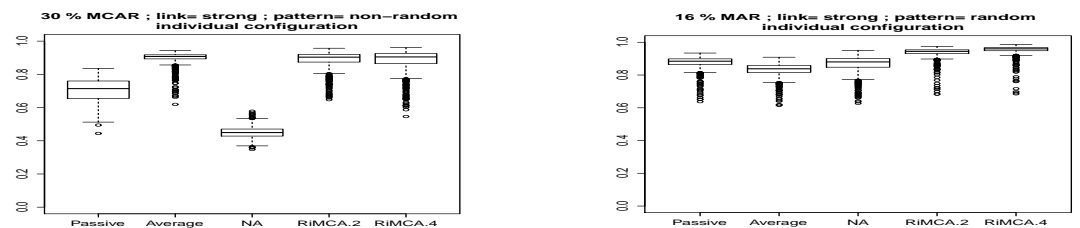

Figure 3: Boxplots of the modified RV coefficients over the 1000 simulations for the case NR, 30\% MCAR, strong, R (left) and 16\% MAR, strong, R (right) 

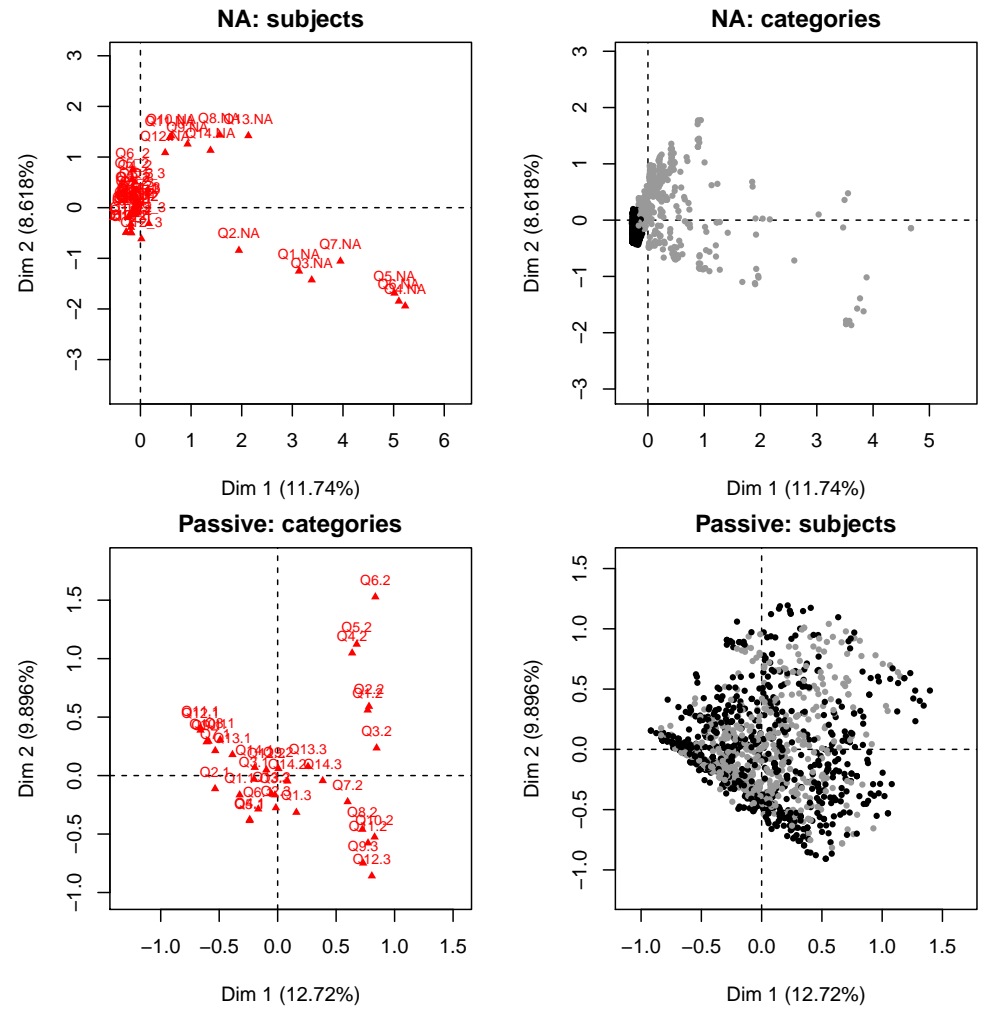

Figure 4: Plot of the categories (left) and the individuals (right) in the principal plane 1-2 obtained by the missing single(NA) method (top) and obtained by the missing passive modified margin (Passive) method (bottom). 

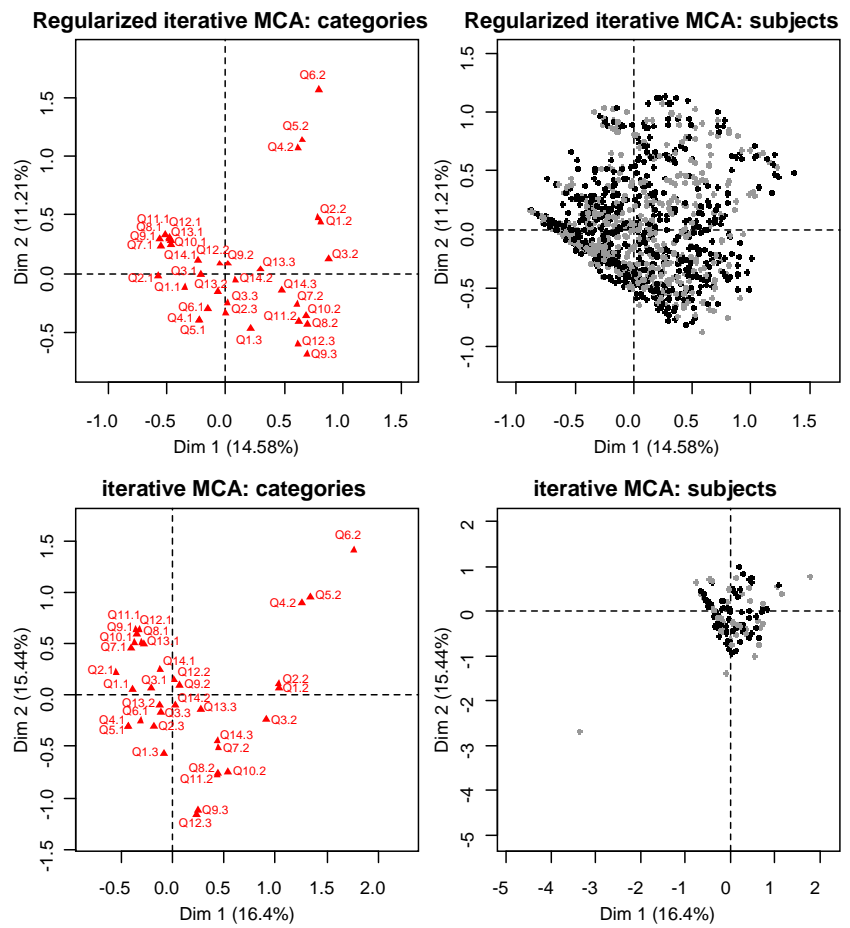

Figure 5: Plot of the categories (left) and the individuals (right) in the principal plane 1-2 obtained by the regularized iterative $M C A$ method (top) and the iterative $M C A$ method (bottom). 